\title{
Coordinating Manipulation in Real-time Interactive Mechanism of College Admission: Agent-Based Simulations
}

\author{
Lan Hou, ${ }^{1}$ Tao Jia, ${ }^{1}$ Xiangbing Wang, ${ }^{2}$ and Tongkui Yu $\mathbb{D}^{1}$ \\ ${ }^{1}$ College of Computer and Information Science, Southwest University, Beibei, Chongqing 400715, China \\ ${ }^{2}$ School of Economics and Management, Guizhou University of Engineering Science, Bijie, Guizhou 551700, China \\ Correspondence should be addressed to Tongkui Yu; ytkui@swu.edu.cn
}

Received 28 March 2020; Revised 5 September 2020; Accepted 19 September 2020; Published 6 October 2020

Academic Editor: Dehua Shen

Copyright $\odot 2020$ Lan Hou et al. This is an open access article distributed under the Creative Commons Attribution License, which permits unrestricted use, distribution, and reproduction in any medium, provided the original work is properly cited.

\begin{abstract}
The matching in college admission is a typical example of applying algorithms in cyberspace to improve the efficiency of the corresponding process in physical space. This paper studies the real-time interactive mechanism (RIM) recently adopted in Inner Mongolia of China, where students can immediately observe the provisional admission results for their applications and are allowed to modify the application before the deadline. Since the universities accept the applications according to the ranking of the scores, RIM is believed to make the competition more transparent. However, students may coordinate to manipulate this mechanism. A high-score student can perform a last-minute change on the university applied, opening a slot for a student with a much lower score. With agent-based simulations, we find that a large portion of students will choose to perform coordinating manipulation, which erodes the welfare and fairness of society. To cope with this issue, we investigate the Multistage RIM (MSRIM), where students with different ranges of scores are given different deadlines for application modification. We find that the multistage policy reduces the chance of manipulation. However, the incentive to conduct manipulation is increased by a higher success rate of manipulation. Hence, the overall social welfare and fairness are further diminished under MS-RIM with a small number of stages, but are improved if the stage number is large.
\end{abstract}

\section{Introduction}

With the development of information and communication technology, the college admission process in the physical space has been transferred to the online information system. Many algorithms, such as deferred acceptance mechanism $[1,2]$, parallel choice mechanism $[3,4]$, and real-time interactive mechanism $[3,5]$, have been designed to reengineer the information process efficiently. The results from the cyberspace are then used to improve the welfare and fairness in the college-student matching of the real world [3-6].

The sequential choice mechanism (SCM) has been used in college admission of China for decades [7]. In this mechanism, each student submits a shortlist of preferred universities. A university will admit students who have listed it as the first choice in the first place. If the university has available quotas after the first round of admission, it will then consider applicants who list it as their second choice and so on. This mechanism is similar to the immediate acceptance mechanism, or Boston mechanism $[8,9]$. In an age of underdeveloped communication technology, this algorithm was widely used due to its simplicity. However, the sequential mechanism has severe limitations: it is not strategy-proof $[2,8]$ and vulnerable to manipulation $[10,11]$. Students need to choose their first choice carefully. Because once a student is rejected by her first-choice university, she will likely be rejected by her second-choice, as the quota of the university may have already been used up. Therefore, students with more information could choose candidate universities more wisely than others. Empirical evidence also shows that a large portion of high-score students failed to be admitted to any colleges only due to inappropriate choices [7].

To fix this problem, a parallel choice mechanism (PCM) has been gradually adopted by most provinces in China since 2008. In this mechanism, students can select several 
"parallel" universities within each choice band. The automated admission information system processes the applications of students from the highest score to the lowest score. Hence, a student will be admitted into the first university in his list which has an unfilled quota. Under this mechanism, all the choices of a high-score student will be considered "parallelly" before moving on to the next student, which greatly increases the chance to be admitted. Empirical evidence also shows that the implementation of the parallel choice algorithm greatly alleviates the problem of students with high test scores not being admitted to any college [4]. Experimental evidence shows that the performance of the parallel choice mechanism is better than the immediate acceptance mechanism [12]. The parallel choice mechanism resembles the deferred acceptance mechanism [2], which is first proposed by Gale and Shapley [1] and widely adopted in two-sided matching $[13,14]$. Deferred acceptance mechanism is proved to have some nice properties such as strategyproof, stability, and elimination of justified envy $[2,13,14]$. The deferred acceptance mechanism asks the students to submit a full preference list of all the colleges. However, in common practice, students are only allowed to submit a list containing a limited number (e.g., 6 in the parallel choice mechanism in some provinces of China) of colleges. Imposing a constrain on the length of the preference lists compels students to adopt a strategic behavior when choosing which ordered list to submit [15] and hinders stable matching [16]. Experimental evidence shows that the students' incentives are drastically affected and more individuals manipulate their preferences under the constrained choice [17]. Chen and Kesten evaluated the sequential, parallel, and deferred acceptance mechanisms by the laboratory experiments. They found that, while the performance of the parallel choice mechanism is better than that of the sequential mechanism, it is worse than that of the deferred acceptance mechanism [12]. It implies that the advantages of the deferred acceptance mechanism are not fully exploited under the parallel choice mechanism due to the limited preference lists.

Inner Mongolia has adopted a novel mechanism called the real-time interactive mechanism (RIM) since 2008, which is expected to alleviate the problem caused by the limited length of the preference list in the parallel choice mechanism. In RIM, students can observe the tentative admission results online and modify their choices at any time before the deadline. This mechanism is similar to the iterative deferred acceptance mechanism [18], which is expected to improve both fairness and social welfare in that it is under perfect information [5]. There is experimental evidence showing that a higher proportion of stable outcomes and truth-telling rates are reached under the iterative deferred acceptance mechanism than the standard deferred acceptance mechanism $[19,20]$.

However, one type of manipulation is possible in the real-time interactive mechanism by the coordination of a high-score student and a low-score student [18]. Because students can modify their applications at any time before the deadline, the high-score student can "hold" a seat in a university and "release" it at the last minute so that the low-score student can take it. The utility of the low-score student is improved because she is admitted by a better university. The high-score student will also be admitted by her truly preferred university due to her score advantages. Hence, their total utility is improved (we assume that the coordinating students have other channels to transfer their utilities in real life and both of them only care about their average utility in our model). However, such coordinating manipulation may erode social welfare and fairness due to distorted outcomes. In practice, the issue of "seat-holding" manipulation has become critical. The authority is aware of it and claims that this behavior will be monitored and prohibited in the new college admission system 2020 [21].

By the approach of agent-based simulations [22, 23], we first study the rationale of the coordinating manipulation under the real-time dynamic mechanism and the impact of coordinating manipulation on social welfare and fairness. We then investigate whether the multistage policy of RIM can alleviate the coordinating manipulation problem. To identify the number of students who would like to take the coordinating manipulation, we use the value at the steady state where both manipulation and nonmanipulation students have no incentive to switch their strategies. We find that a considerable portion of students will take the coordinating manipulation, and both the welfare and fairness of the society are lower than the situation when there is no manipulation. Furthermore, we propose a variant of RIM with multiple stages (MS-RIM), where students with different scores have different deadlines to submit their applications, with higher score students earlier and lower score students later. Simulations show that the multistage policy has complex effects on matching results of RIM. The strategy can reduce the chance of coordinating because of the different deadlines to submit applications. However, it amplifies the students' incentive to conduct coordinating manipulation due to the scarcity of manipulation opportunity and consequent higher reward. Moreover, it helps to avoid the congestion that all manipulation students transfer their seats almost simultaneously and increases the success rate of the manipulation. The combination of the three effects indicates that when the number of stages is small, social welfare and fairness are diminished by the increased successful manipulations. Only when the number of stages is sufficiently large, the successful manipulation number decreases and the social welfare and fairness are improved.

The organization of this paper is as follows. Section 2 describes the background of this experiment. Section 3 introduces the agent-based model of the real-time dynamic mechanism of college admission. Section 4 presents and discusses the results and then comes to conclusions in Section 5.

\section{Background}

2.1. Real-Time Interactive Mechanism. The real-time interactive mechanism was firstly trialed in the college application and admission process of Inner Mongolia for a portion of the students in 2006 and 2007. It was then applied to all students since 2008, with a few modifications and improvements in the following years. 
Before the application and admission process, the universities submit their enrolment plans and are assigned some quotas, and the students take the college entrance examination and obtain their scores, which is the main criteria of the admission. The number of students with different scores is publicly available, as well as the universities' enrolment plans and their lowest scores of admitted students in the previous years. The students are advised to well recognize the rank of their score among all students and choose some preferred and probably achievable universities.

When the admission process starts, every student needs to $\log$ in to the online admission system and choose a university to apply. On receiving a student's new application, the computerized system will insert this student to the ranking list of all students that apply to the same university according to their scores in the descending order. A student can see her position on the list and roughly know if she will be admitted immediately after submitting the application. The cut-off score, the minimum score of those students within the enrolment quotas of a school, will be updated in real-time and made public regularly.

At any time before the system closes, the students can check if they could be admitted and decides whether to make a new application. Students can modify their choices as many times as possible before the deadline.

When the system is closed, the students whose score is ranked within the quota of their applied university will be officially admitted. Otherwise, they will be rejected by this university and cannot be admitted by other universities too.

The advantage of the real-time interactive mechanism is information transparency. A student can observe whether he could be admitted by the applied university immediately after the application submission, and this enables students to apply the universities both preferred and achievable, which is impossible under other mechanisms.

\subsection{Coordinating Manipulation of High- and Low-Score}

Students. The real-time interactive mechanism is not perfect. One shortcoming of this mechanism is that it is vulnerable to a type of manipulation by the coordination of a student with a high exam score and another student with a low exam score.

At the beginning of the admission procedure, the highscore student does not apply to his favorite university, but applies to the university that the low-score student prefers. This means that the high-score student "holds a seat" from a university for the low-score student. In the last few minutes before the deadline, the high-score student can "release this seat" so that the low-score student can apply for this university before other students notice this vacancy. After releasing a seat, the high-score student immediately applies to his truly preferred university. As a result, the high-score student can be accepted because of the advantage of his score, and the low-score student can be admitted by a better university due to a successful manipulation.

Although a successful manipulation can benefit the two manipulation students, this may make unfairness to other students and welfare loss to society. If there were no manipulation, another student with a score between the high-score and low-score student can be admitted by the university that admits the low-score student. Moreover, the high-score manipulation student switches to a university at the last minute before the deadline, and this usually pushes another student out of the enrolment quota of the applied university without the chance to apply to other universities due to the limited time.

2.3. Multistage Real-Time Interactive Mechanism. Since 2008, the admission process is carried out by multiple stages, which we called the multistage real-time interactive mechanism (MS-RIM). Students with different scores are given different deadlines to submit their applications, and the deadline for students with higher scores is earlier than those with lower scores. For example, students whose scores range from 600 to 629 need to submit their applications before $4 \mathrm{pm}$, and students whose scores range from 570 to 599 submit their applications before $5 \mathrm{pm}$, and so forth.

This multistage policy is introduced to tackle the network congestion problem because many students modify their applications just before the deadline. Especially, when a high-score student switches to a university, some low-score students who are ranked lower near the cut-off score will be afraid of being refused by this university and switch to apply to other universities, and they will then push other students to switch universities too. The multistage policy provides students with different scores different deadlines, and this alleviates the number of switches and improves the problem of network congestion.

It is still unclear how the multistage policy influences coordinating manipulation. Because the deadline to submit applications varies for students with different scores, some of them will have no opportunity to conduct the coordinating manipulations. However, coordinating manipulations remains a big issue in practice despite the implementation of the multistage policy for many years.

We conduct simulations to confirm that, although the multistage policy can really reduce the occurrence of the coordinating manipulation, it increases the incentive of students to manipulate the mechanism.

\section{Methods}

We study the college application and admission process under real-time interactive mechanism by agent-based simulations, which provide an ideal tool to model complex adaptive systems by a bottom-up approach [22, 23]. By modeling the behaviors of the individual students, we can observe how the interactions self-interested individuals build up the macrolevel phenomena such as the welfare and fairness of the resultant matching. An agent-based simulation is also flexible to perform scenario analysis which is usually necessary for practical policy making. This approach has been adopted to study the college matching problem $[24,25]$.

The simulation model settings are initialized as follows:

(1) A set of students $N=\{1,2, \ldots, n\}$ whose exam scores are randomly generated from the uniform distribution from score_min to score_max. 
(2) A set of universities is denoted by $S=\left\{s_{1}, s_{2}, \ldots, s_{m}\right\}$.

(3) The enrolment quotas of the universities are denoted by $Q=\left\{q_{1}, q_{2}, \ldots, q_{m}\right\}$, which means that the number of students that university $i$ will admit is $q_{i}$.

(4) The preferences of students over universities are denoted by $P=\left\{P_{1}, P_{2}, \ldots, P_{n}\right\}$, where $P_{i}$ is a permutation of all universities, for example, $P_{i}: s_{1}$, $s_{2}, s_{3}, \ldots, s_{m}$, which means that, for student $i$, university $s_{1}$ is his first choice, university $s_{2}$ his second choice, university $s_{3}$ his third choice, and so forth. We assume there is a public ranking of universities, such as the Best Global Universities Ranking by U.S. News and World Report. The student preference $P$ is randomly generated by disturbing the public ranking, which means that students may have different preferences over universities, but in general, top-ranked universities are preferred by more students than low-ranked ones.

(5) The preferences of universities over students are denoted by $R=\left\{R_{1}, R_{2}, \ldots, R_{m}\right\}$. In this model, all universities' preferences over the students are completely based on their scores.

(6) The minimum score difference between the two manipulation students is score_diff. Since the highscore student will hold a seat for the low-score student in the coordinating manipulation, only the score difference between them is larger than score_diff, and then they can take the manipulation strategy.

(7) The proportion of students who attempt to find partners to take coordinating manipulation is denoted by $\rho$, which is called the manipulationseeking ratio. In the simulation, a proportion $\rho$ of the students are randomly selected as manipulationseeking students. Each manipulation-seeking student has one chance to find a partner. For a manipulation-seeking student who has no partner (student A, for example), he will ask another student (say student B) which is randomly chosen from all other students. If $B$ is also a manipulationseeking student and has no partner, and their score difference is larger than score_diff, they will become partners and are ready to conduct the coordinating manipulation, and student $B$ will not search for other partners anymore; otherwise, student A cannot conduct manipulation although he is willing to do so. Those who find partners to conduct coordinating manipulation are called manipulation students.

(8) The number of rounds in one simulation is $T$. The simulation runs in discrete steps, and every student has one chance to update his application at each round.

(9) The round when the high-score manipulation students release the seat that they hold for the low-score manipulation students is $t_{\text {seat-transfer }}$ We simulate with different $t_{\text {seat-transfer }}$ and the students will adapt to choose the optimal $t^{*}$ seat-transfer to maximize their utilities.

(10) To simulate the situation that some students may fail to submit their applications due to the operation failure (such as network congestion, slow system response, and operation error), each student has a probability $\lambda_{t}$ that his submission is unsuccessful. The failure probability varies with the round number $t$, and the closer the round number is to the deadline, the larger $\lambda_{t}$ will be.

The simulation procedure is described as follows. The admission process runs for $T$ rounds. In each round, every student has one chance to submit his application. The order of students to submit applications is rearranged randomly for each round. Before submitting applications, every student can inquire the information about university's quotas and cut-off scores of universities. The nonmanipulation students will report their true preferences, that is, they will apply to their most preferred university among all feasible universities whose quota is not filled or the applicant's score is higher than the last one in the university's application list. For manipulation students, the high-score manipulation student will apply for the most preferred feasible university of low score, and the low-score manipulation student will apply according to his true preferences before the seattransfer time $t_{\text {seat-transfer }}$. At round $t_{\text {seat-transfer }}$, the high-score student releases the seat of the university that the low-score student preferred and then applies for his truly preferred feasible school, and at the same time the low-score student applies for this "released seat" immediately. All the operations of the students are vulnerable to failure with probability $\lambda_{t}$. The ranking of students in the university's application list is updated in the descending order as soon as a new application is submitted. After all students have submitted their applications, each student can observe the temporary matching result and know if their ranking is within the quota of the universities. Then, a new round starts and all students can modify their applications in the new round.

The admission process ends after Trounds of simulation, and the students whose scores are within the quotas of the applied universities are officially admitted. Then, the utilities of students and universities can be calculated according to the admission results and their preferences.

A student's utility is determined by the position of the university that admitted him in his preference. For example, when there are $m$ schools, the utility of a student is $m / m=1$ if he is admitted by his first-choice university and is $(m-1) / m$ if admitted by the second-choice university and so forth, and if he is admitted by the last preferred university, his utility is $1 / \mathrm{m}$. Certainly, the utility of unmatched students is zero.

The utility of universities can be calculated in the same manner. When there are nstudents, a university's utility from admitting the student with the highest score is $n / n=1$ and is $(n-1) / n$ from admitting the second-highest score student and so forth. Since each university may accept more 
than one, the total utility of a university is the sum of the utilities from all admitted students. If a university failed to enrol enough students, the utility from the vacant seat is 0 . Then, the utility of a university can be adjusted by dividing the total utility with its quota $q$.

The parameter settings of our simulations are $n=450$, $m=30, \quad q=15, \quad$ score_min $=550, \quad$ score_max $=700$, score_diff $=20, \rho$ ranges from 0 to $1, T=100, t_{\text {seat-transfer }}$ ranges from 1 to $T$, and $\lambda_{t}=t / 1000$, and $t=1$ to 100 . Although, we set the max round number of one simulation as 100 , the system stabilizes at about the 35 th round.

\section{Results and Discussion}

4.1. Coordinating Manipulations at Steady State. Although every student can choose to coordinate with other students to manipulate the mechanism, it does not imply that all of them are willing to do so. As rational agents, they will make their choices according to the expected utility from different strategies. If the expected utility from manipulation is larger than that from nonmanipulation, more students will choose manipulation, otherwise, more students will adapt to nonmanipulation. Then, a steady state where the manipulation students and nonmanipulation students obtain the same utilities characterized the outcome of the manipulation game. At the steady state, the percentage of the manipulation and nonmanipulation students will not change despite that the self-interested students are free to make their choices.

There are a proportion $\rho$ of the students (about $\rho n$ ) who are willing to find partners to conduct manipulation, and about $(1-\rho) n$ students truthfully submit their applications. Among manipulation-seeking students, some of them can successfully find their partners, while others cannot. This is simulated by randomly matching them in pairs, and if the difference between the score of two partners is larger than score_diff, they can really conduct the coordinating manipulation. So, only the students who are willing to conduct manipulation as well as have successfully found the partners are called manipulation students. The students who do not really conduct manipulation are called nonmanipulation students, including those who are willing to manipulate but fail to find the partners.

To find the steady state, we simulate with different $\rho$ that ranges from 0 to 1 and calculate the average utility of the manipulation students and nonmanipulation students, respectively, under different values of $\rho$. Some typical results are shown in Figure 1.

Since high-score and low-score students coordinate, we assume they can transfer utilities to each other by other channels in real life, and hence they can be regarded as a coalition and both of them make their decision according to their average utilities, as indicated by the red lines marked with circles.

One decision that the manipulation students need to make is when the high-score students will transfer the seat to the low-score students. If the seat-transfer happens too late, they will take the risk of application failure at the last minute due to network congestion or other issues. If they transfer too early, the low-score students may be pushed out of the list of their preferred university by other students who apply to this school in the following rounds. Hence, they will choose the optimal round to release the seats. In the simulation, we try all seat-transfer time and calculate the corresponding average utilities of the manipulation students; then, the seat-transfer time that provides the largest utility of manipulation students will be their optimal choice, for example, the 1st round before deadline when $\rho=0.01$ in Figure 1(a) and the 10th when $\rho=0.1$ and 11th when $\rho=0.3$ in Figure 1(b) and 1(c). In Figures 1(e) and 1(f), the utilities of manipulation students are always lower than nonmanipulation students whenever they release the seats, so they will not choose to coordinate. In particular, we find that the utility difference between manipulation students and nonmanipulation students stabilizes at about the 15th round, so all figures shown in this paper display the results from the last round to 15 th round.

Another decision of the students, on a higher level, is whether to conduct coordinating manipulation. This actually depends on how many students are doing so. When there are few students who attempt to coordinate, such as $\rho=0.01,0.1$, and 0.3 in Figures $1(\mathrm{a})-1(\mathrm{c})$, the utility of the manipulation students (at the optimal seat-transfer time) will be larger than that of nonmanipulation students; this is because the information provided in the tentative admission result is mostly real, and there are few switches of applied university of manipulation students before the deadline. Hence, the nonmanipulation students may learn to adopt the manipulation strategy for higher utility and the portion of the manipulation-seeking students $(\rho)$ will increase.

However, if there are too many manipulation-seeking students, such as $\rho=0.7$ and 0.9 in Figures $1(\mathrm{e})$ and 1(f), the utility of nonmanipulation students is slightly larger than manipulation students; this is because the information of the tentative admission result is not reliable and there are too many application switches before deadline. Hence, some manipulation students will choose not to take manipulation strategy even though they have the chance to do so, and the portion of manipulation-seeking students $(\rho)$ will decrease.

As a result, the system will stabilize at a steady state where the utility of manipulation students is equal to that of the nonmanipulation students, at $\rho=0.5$ in Figure $1(\mathrm{~d})$, and no manipulation students want to switch to nonmanipulation students and vice versa. Figure 2 provides the relationship between manipulation-seeking ratio $(\rho)$ and the utilities of different students with more values of $\rho$, and we can see the steady state at $\rho^{*}=0.5$, with the utility of manipulation students larger than that of nonmanipulation students when $\rho<\rho^{*}$ and smaller when $\rho>\rho^{*}$. Therefore, the consequence of the students' rational choices is that about half of the students will try to find partners to conduct coordinating manipulation. Here comes Result 1.

Result 1. A considerable portion of students will conduct the coordinating manipulation under the real-time interactive mechanism.

Next, we will investigate how the manipulation impacts the social welfare and fairness of the matching by real-time 


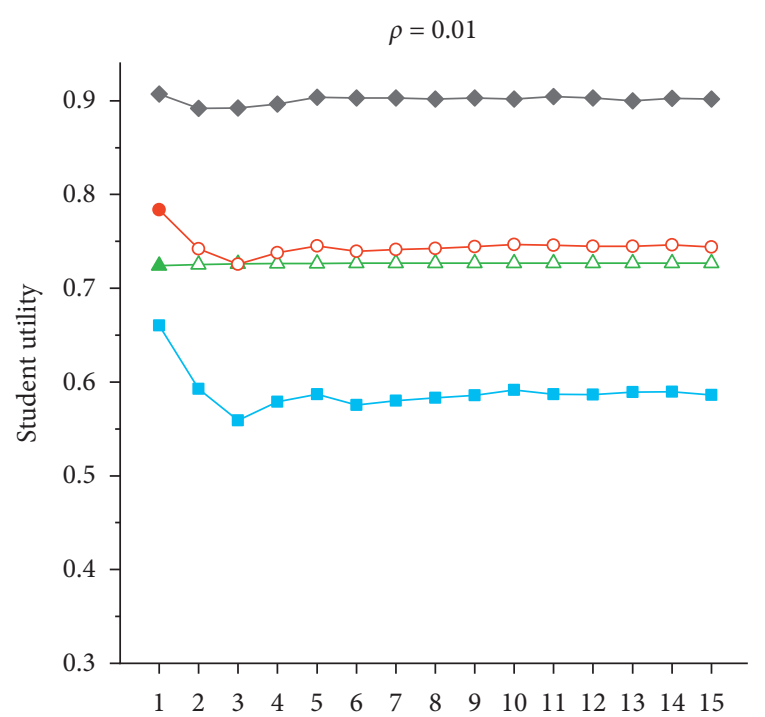

Seat-transfer time (counting backward from deadline)

$\smile$ High-score manipulation students

- - Low-score manipulation students

- - Manipulation students

$\triangle-$ Nonmanipulation students

(a)

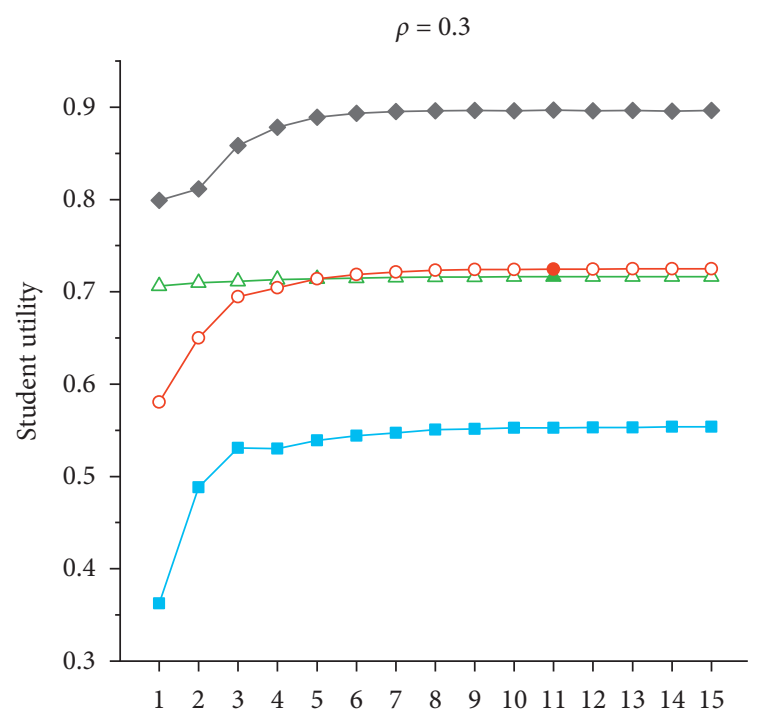

Seat-transfer time (counting backward from deadline)

$\smile$ High-score manipulation students

-1- Low-score manipulation students

- - Manipulation students

$\triangle$ Nonmanipulation students

(c)

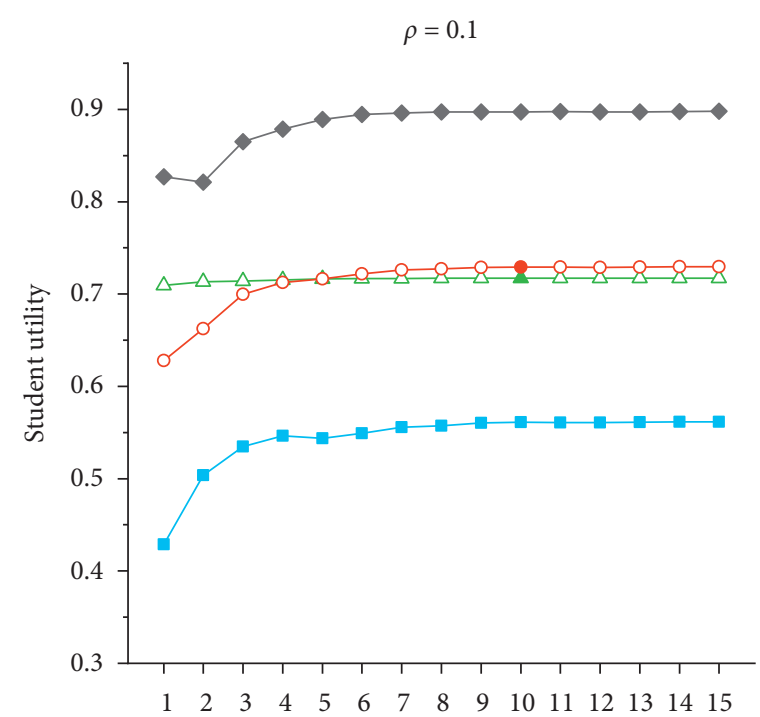

Seat-transfer time (counting backward from deadline)

$\smile$ High-score manipulation students

$\rightarrow$ Low-score manipulation students

- - Manipulation students

$\triangle-$ Nonmanipulation students

(b)

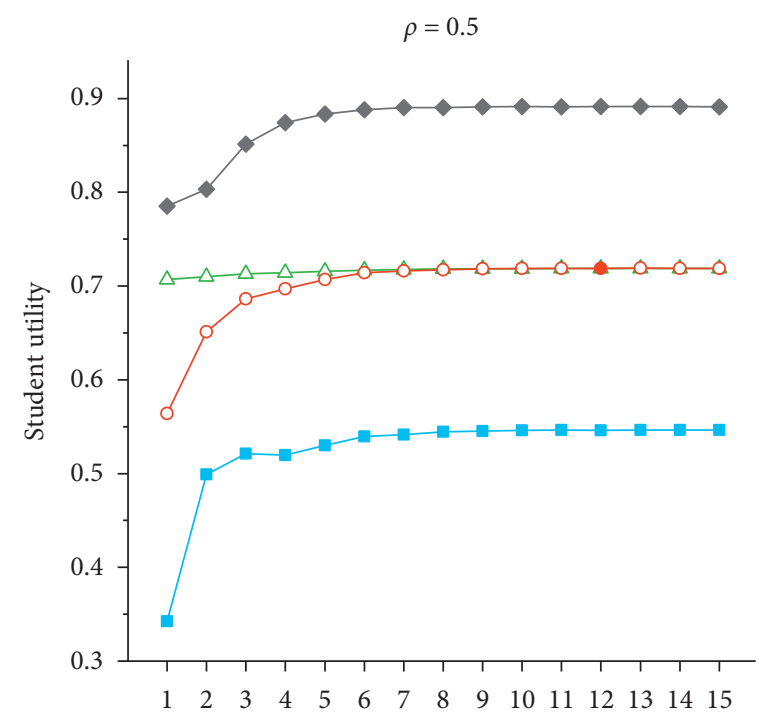

Seat-transfer time (counting backward from deadline)
$\checkmark$ High-score manipulation students
- - Low-score manipulation students
- - Manipulation students
$\triangle \quad$ Nonmanipulation students

(d)

Figure 1: Continued. 


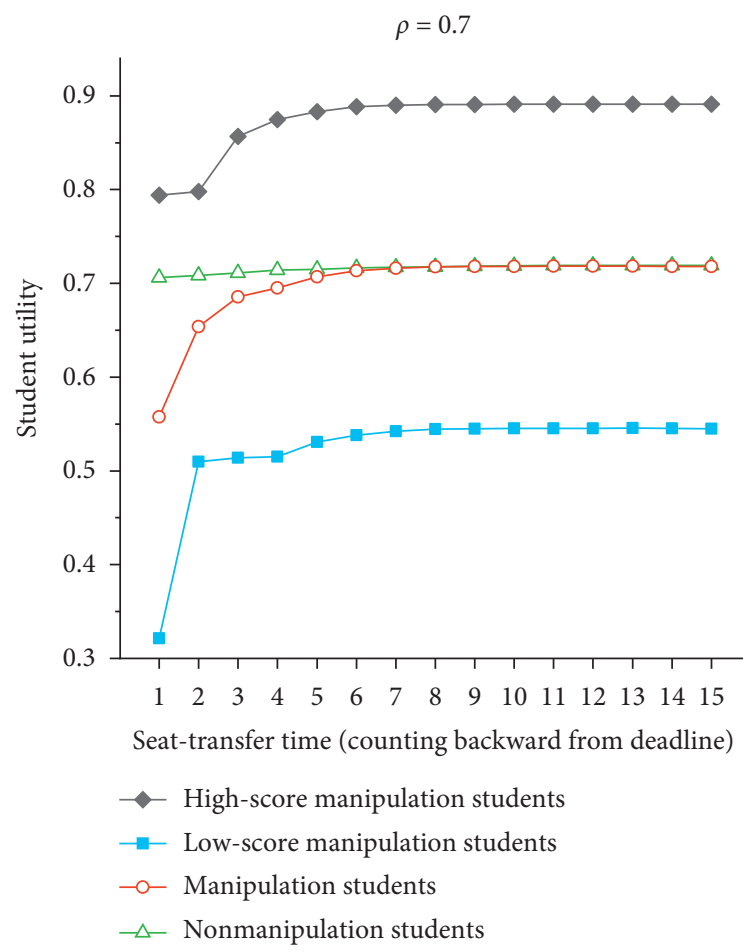

(e)

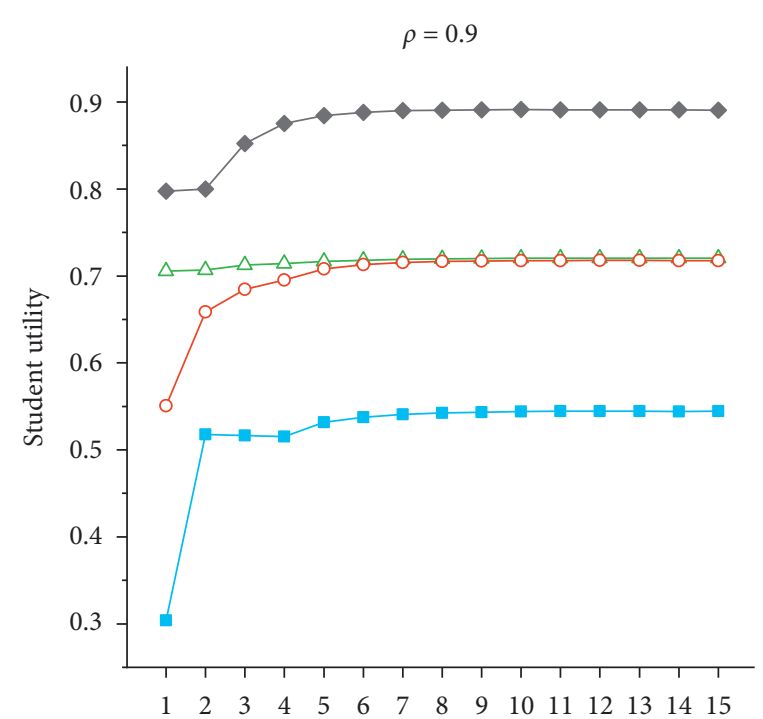

Seat-transfer time (counting backward from deadline)

$$
\begin{aligned}
& -\_ \text {High-score manipulation students } \\
& -\_ \text {Low-score manipulation students } \\
& -\_ \text {Manipulation students } \\
& \rightarrow-\text { Nonmanipulation students }
\end{aligned}
$$

(f)

Figure 1: Utilities of manipulation and nonmanipulation students under different manipulation-seeking student ratio $\rho$. The $x$-axis represents the time when the high-score manipulation student transfers the seat to the low-score manipulation student, and it is the round number counted backward from the deadline. The $y$-axis represents the average utilities of students. The black line marked with diamonds is the average utility of the high-score manipulation students, the blue line marked with squares is for low-score manipulation students, the red line with circles is the average utility of all the manipulation students (both high- and low-score manipulation students), and the green line with triangle is for nonmanipulation students. The solid marks represent the optimal transfer time $t^{*}{ }_{\text {seat-transfer }}$ of manipulation students. For each parameter setting, we run the simulation for 100 times and calculate the average utilities.

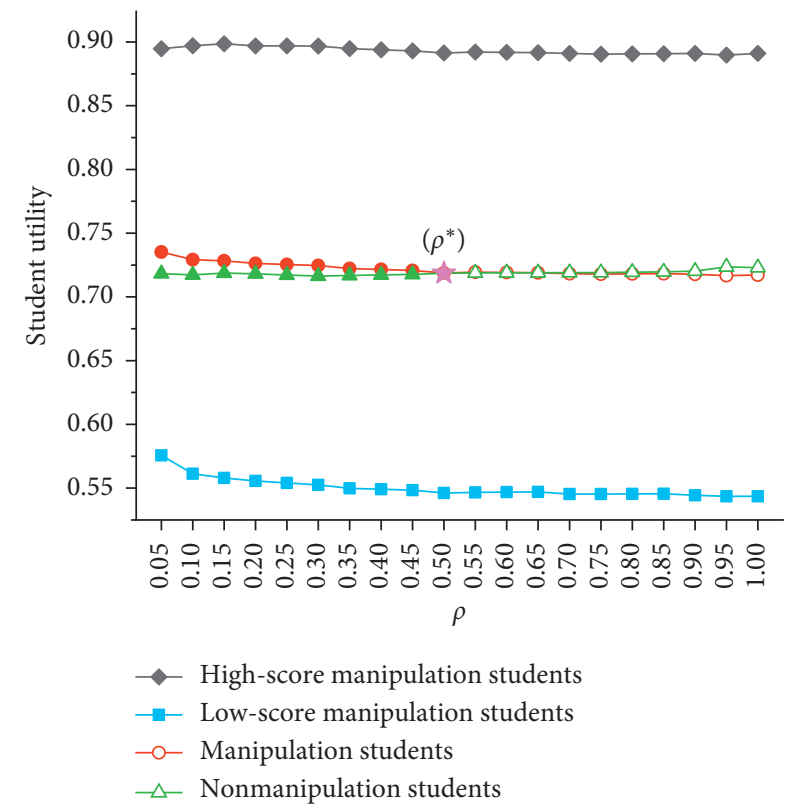

FIGURE 2: Relationship of manipulation-seeking ratio $(\rho)$ and the utilities of different students. The utilities of students are calculated with the optimal seat-transfer time of manipulation students. The steady state $\left(\rho^{*}\right)$ is marked with pentagram. interactive mechanism. Social welfare is measured by the utility per capita for all students and all universities. We calculate the average utility of all students and that of all universities for $\rho=0$ (no manipulation) and $\rho=0.5$ (the steady state with manipulation). We can see that the average utility of all students from 0.7199 (no manipulation) to 0.7187 (steady state with manipulation), as shown in Figure 3(a). To investigate how the manipulation and nonmanipulation students with different scores are affected by the coordinating manipulation, we calculate the average utility of the top-ranked (with scores higher than the mean score of all students) and low-ranked (with scores lower than the mean score) manipulation and nonmanipulation students, as shown in Figures 3(b) and 3(c). The average utility of both the top- and low-ranked nonmanipulation students becomes smaller. The reason that the utility of top-ranked nonmanipulation students becomes worse off is that the high-score manipulation students will resubmit their applications after releasing the seat for the low-score manipulation student, and they will push some of the high-score nonmanipulation students out of the admission list of the applied university, then cause them to have no time to resubmit applications. For low-ranked nonmanipulation students, in addition to the above reason, it is also because some of the seats are held by the manipulation students and 


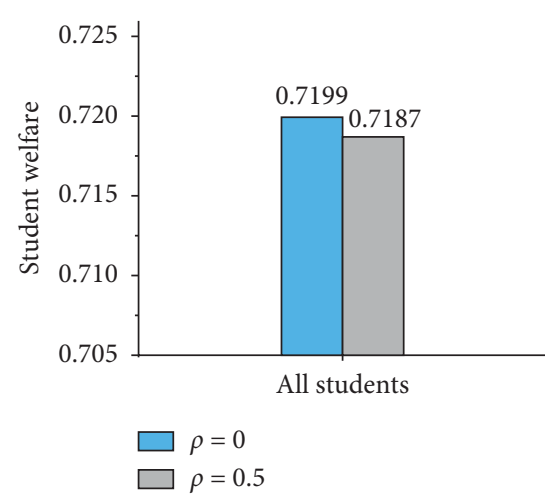

(a)

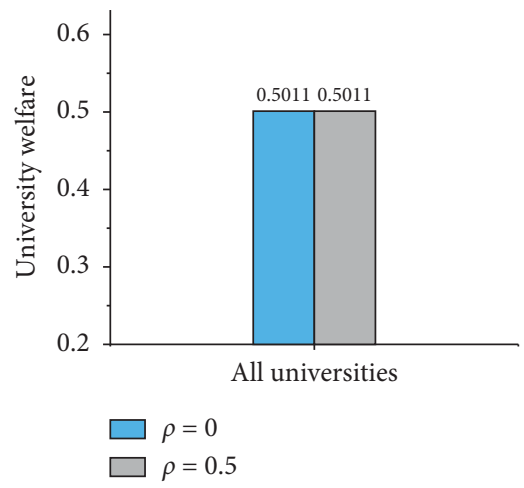

(d)

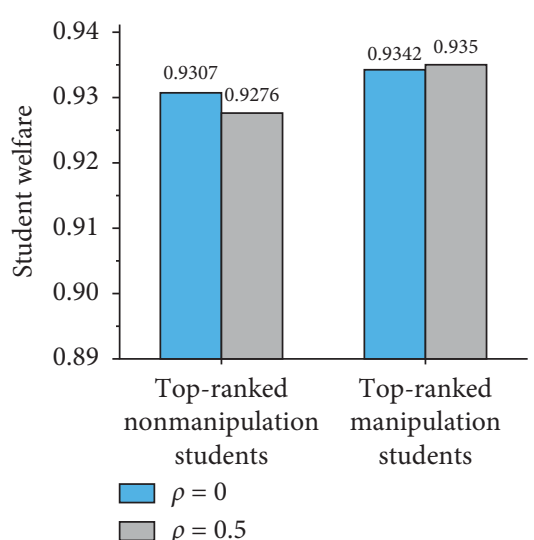

(b)

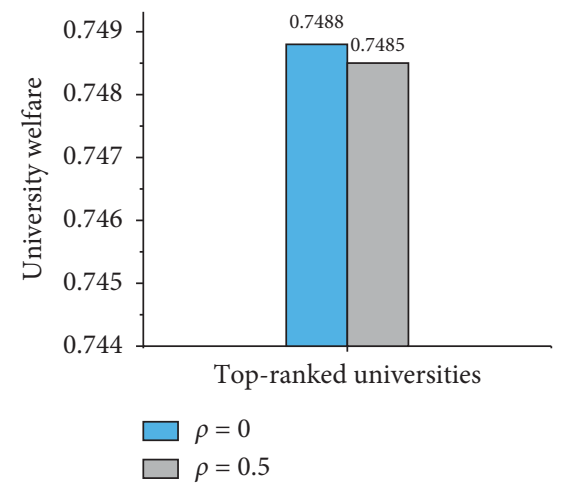

(e)

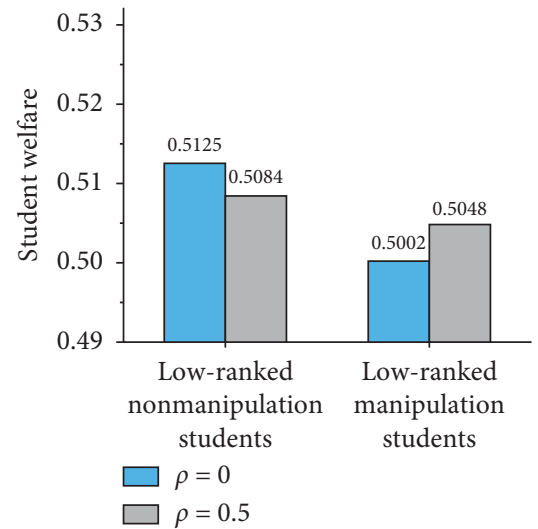

(c)

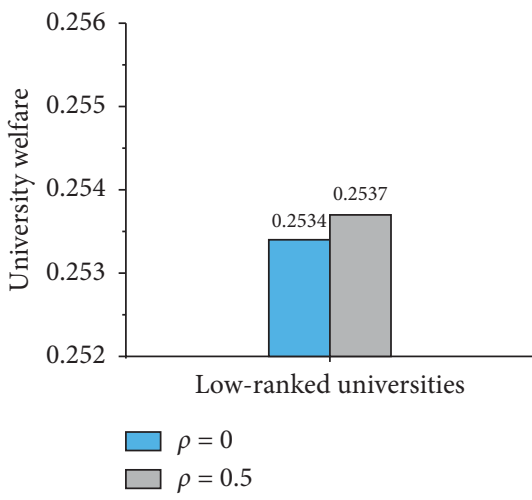

(f)

Figure 3: Student welfare and university welfare under the state with no manipulation $(\rho=0)$ and under the steady state with manipulation $(\rho=0.5)$. The top-ranked and low-ranked students are those whose scores are ranging from 625 to 700 and 500 to 624 . The top-ranked and low-ranked universities are No. 1-15 and No. 16-30 universities in the public ranking, respectively.

the cut-off score of the university is raised, so they can only choose their less preferred universities.

The average utility of all universities for $\rho=0$ (no manipulation) is the same as that for $\rho=0.5$ (the steady state with manipulation), as shown in Figure 3(d). This is because all universities have the same preferences over students (all prefer students with a higher score), and the total utility of all schools does not change under any college-student matching as long as the quotas of all universities are fulfilled. However, the utility distribution among the universities is different, with the utility of the top-ranked universities (No. 1-15 in the public ranking) damaged while that of the lower-ranked universities (No. 16-30) improved, as indicated in Figures 3(e) and 3(f). This is because the low-score manipulation students can be admitted by better ranked universities through successful coordinating manipulation, which causes the top-ranked universities to accept low-score students and their utilities are damaged. On the contrary, there must be increases in utilities of low-ranked universities.

The fairness of a college-student matching is measured as follows. For each student, he can observe the cut-off scores (the minimum score of all admitted students) of all universities that he preferred but do not admit him, and if his score is larger than any one of the cut-off scores, he will feel unfair; otherwise, he will feel fair. Hence, the fairness index is defined as the proportion of students who feel fair among all students. Figure 4 compares the fairness of the situations with and without manipulations. When there is no manipulation, the property of no justified envy of deferred acceptance mechanism assures that there is no unfairness [2], which is consistent with the simulation results that the fairness index equals 1 . However, at the steady state with manipulation, the fairness index declines to 0.9456 , which implies that about $5 \%$ of students feel unfair. Hence, we have Result 2 as follows.

Result 2. The social welfare and fairness of the matching are both damaged under real-time interactive mechanism due to the manipulation behaviors.

4.2. Manipulation under MS-RIM. In this section, we investigate the manipulation behavior under the multistage RIM. The admission procedure runs for $K$ stages, and each stage runs for Trounds. After the end of stage 1, the students with scores ranging from score_min + (score_max-score_$\min )(K-1) / K$ to score_max cannot submit their applications anymore. Similarly, students with scores ranging from score_min $+($ score_max-score_min $) \quad(K-2) / K$ 


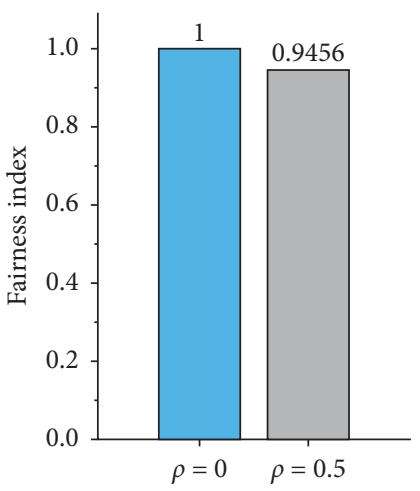

FIGURE 4: Fairness index under the state with no manipulation $(\rho=0)$ and the steady state with manipulation $(\rho=0.5)$.

score_min + (score_max-score_min $)(K-1) / K$ cannot submit their applications any more after the end of stage 2 and so on. The admission system closes after the last stage.

For each stage of an MS-RIM, we need to find the steady state $\left(\rho^{*}\right)$ where the proportion of manipulation-seeking students does not change. Similar to the previous section, we simulate with different values of $\rho$ ranging from 0 to 1 and choose the one that provides the same utility for both the manipulation and nonmanipulation students. Given the value of $\rho$, the utilities of students also depend on the seattransfer time $\left(t_{\text {seat-transfer }}\right)$ of manipulation students, that is, when the high-score students transfer seats to low-score students. As rational individuals, they will choose the optimal time that provides the maximum utility. Hence, for a given value of $\rho$, we simulate all seat-transfer time and choose the one that provides the highest utility for manipulation students, as indicated by the solid marks. We calculate the steady state stage by stage, and the manipulation-seeking ratio at the steady state may vary across stages.

For two-stage RIM, we firstly consider the decision of students in the 1st stage, whose scores are between 625 and 700 . When few students attempt to coordinate $(\rho=0.1)$, the utility of the manipulation students (at the optimal seattransfer time) will be larger than that of nonmanipulation students, as illustrated in Figure 5(a). It is expected that more students will adopt this strategy. When all students are willing to find partners to conduct coordinating manipulation $(\rho=1)$, the utility of manipulation students is still larger than nonmanipulation students, as in Figure 5(b). This implies that the steady state is $\rho^{*}=1$ and all students are willing to conduct the coordinating manipulation eventually as the consequence of the rational choice of students. Given that the students in the first stage behave as the steady state indicates $\left(\rho^{*}=1\right.$ in this simulation), the steady state for the second stage (with students' scores from 550-624) can be obtained similarly by simulating with different values of $\rho$. And it is found that the steady state is also $\rho^{*}=1$ because the utility of manipulation students is always larger than that of the nonmanipulation students for all $\rho$ values, as illustrated by Figures 5(c) and 5(d).

For three-stage RIM, by the same method, we find that the steady state is $\rho^{*}=1$ for all stages, implying that all students are willing to conduct coordinating manipulation due to the higher utility than nonmanipulation, as illustrated by the typical results in Figure 6 . Two new observations should be noticed. The first is that the utility difference between the manipulation and nonmanipulation students at a steady state is larger in late stages than in the early stages. Certainly, the larger is the difference, the stronger incentive to conduct manipulation that the students will have, that is, the incentive to conduct manipulation is larger in the late stage than in the early stage. The second observation is that the optimal seat-transfer time $\left(t^{*}\right.$ seat-transfer $)$, when the highscore manipulation student releases the seat for the lowscore student, in the 3rd stage of three-stage RIM is the last round before the deadline, that is, compared with manipulation in the early stage, the manipulation students in late stages transfer their seats at the time closer to the deadline. This is because the low-score manipulation students in this stage have the least scores (550-580), and they can only be accepted by the lower-ranked universities even if there was no manipulation. This implies that their "opportunity cost" is low, and they would like to take the risk to improve their utilities by transferring the seat at the last round.

To investigate the observations discussed above and how the multistage policy affects matching results of RIM, we also simulate the four-stage, five-stage, and six-stage RIM, and all the results are summarized in Table 1 (other parameters are the same as above).

Simulation results averaged for 100 runs. The ratio of students who are willing to conduct coordinating manipulation at the steady state is $\rho^{*} ; t^{*}$ seat-transfer is the optimal time for the manipulation students to transfer the seat from the high-score student to the low-score student; utility difference is the average utility of the manipulation students minus the average utility of the nonmanipulation students; the manipulation number is the number of students that find partners to conduct manipulation (that is, the scores' difference of the two paired student is larger than the critical value score_diff); the successful manipulation number is the number of manipulation students who successfully transfer the seat from the high-score student to the low-score students and do not need to be pushed out by other students in the following rounds; student (university) welfare is the average utility of all students (universities); the fairness index is the ratio of students who do not feel unfair.

The steady states are $\rho^{*}=1$ for each stage of all versions of RIM except the one-stage version (the third column of Table 1). This means that all of the students are trying to find partners to conduct coordinating manipulation and the utility from the manipulation is always larger than nonmanipulation under MS-RIM. Hence, we have the following result.

Result 3. Students have a stronger incentive to conduct coordinating manipulation under MS-RIM than one-stage RIM.

The explanation for the result is that the benefit of the manipulation is smaller when there are more students to conduct coordinating manipulation. This is because the information on the tentative admission result is not reliable when most students report false preferences. Meanwhile, the 


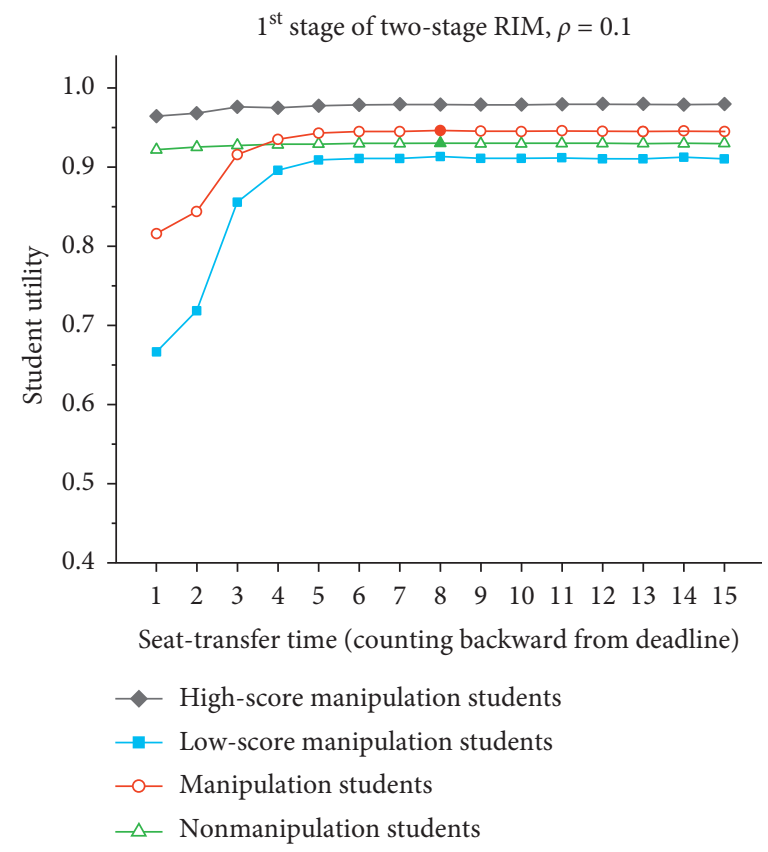

(a)

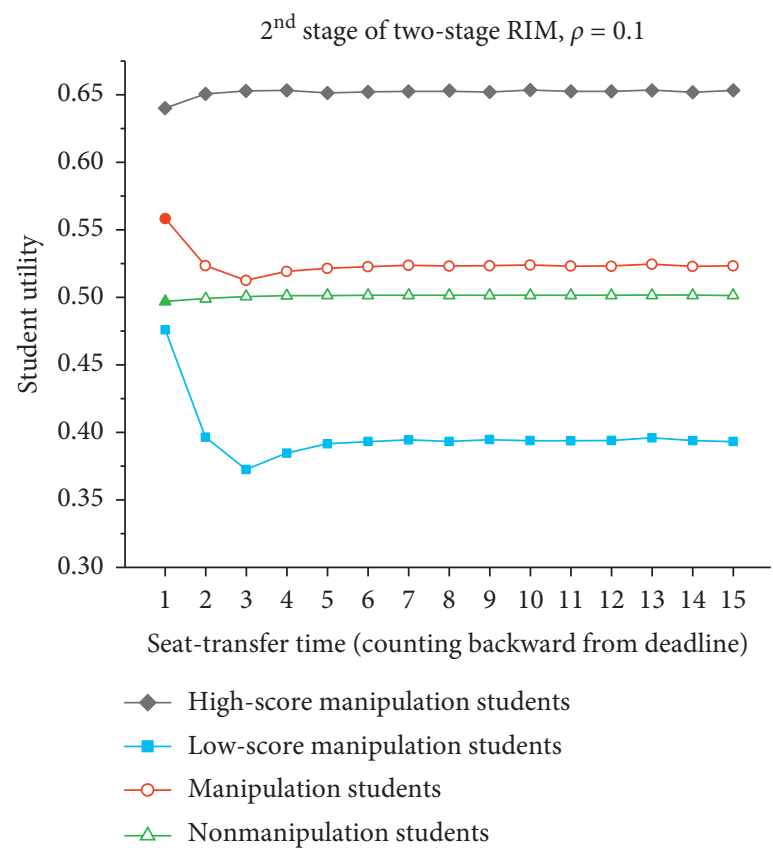

(c)

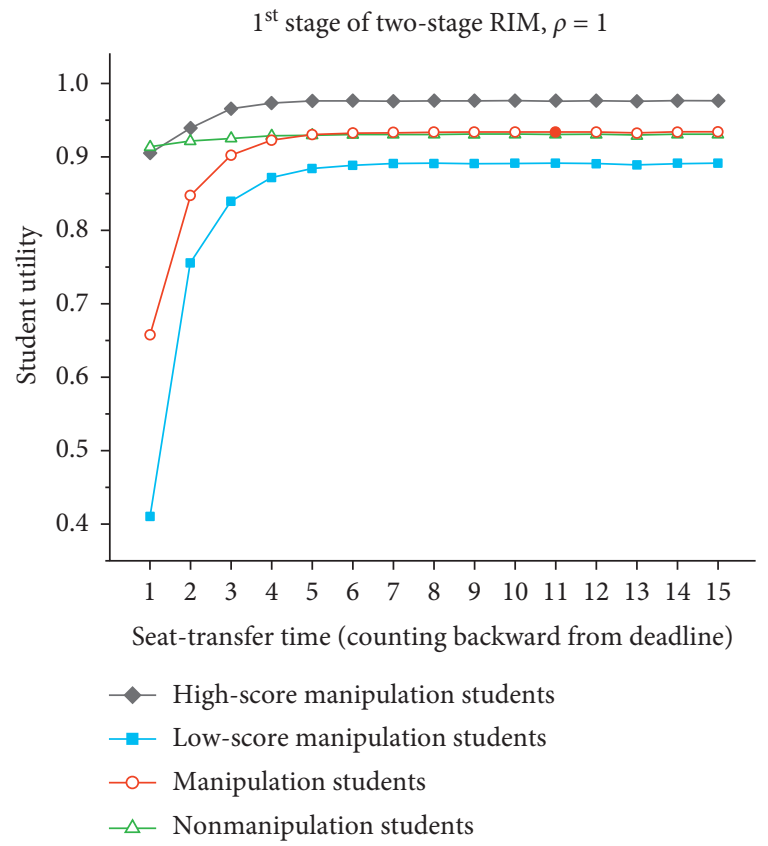

(b)

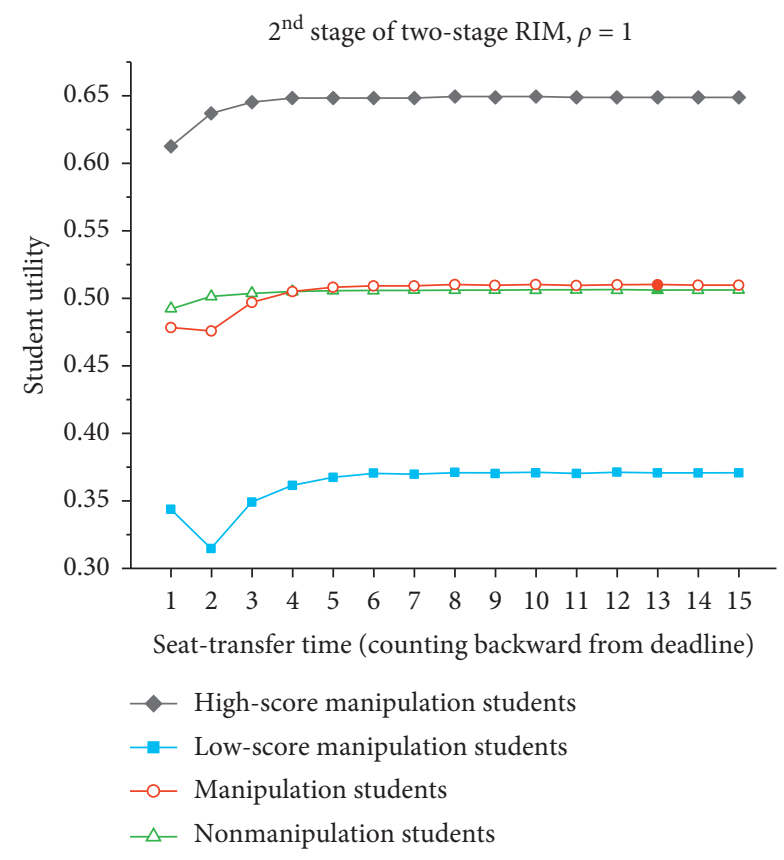

(d)

FIGURE 5: Utilities of manipulation and nonmanipulation students under different manipulation-seeking ratios $\rho$ under two-stage of RIM. The utility of manipulation students is always larger than that of nonmanipulation students for different values; hence, the steady state is that all students are willing to conduct coordinating manipulation.

seat-transferring of the coordinating students may affect each other, for example, when a high-score student releases the seat for his partner and chooses his preferred university, another student who just applied this university may be pushed out of the admission list and he will immediately resubmit a new university then continuously pushing the other one out of the admission list, that is, there are too many application switches before the deadline. Under the multistage policy, students in different stages cannot coordinate; hence, the number of the students who can conduct coordinating manipulation becomes smaller, and the scarcity of the manipulation makes it more beneficial and therefore, the students are more willing to take this behavior.

We can also see that the utility difference between manipulation and nonmanipulation students is generally larger for late stages than early stages in a certain version of 


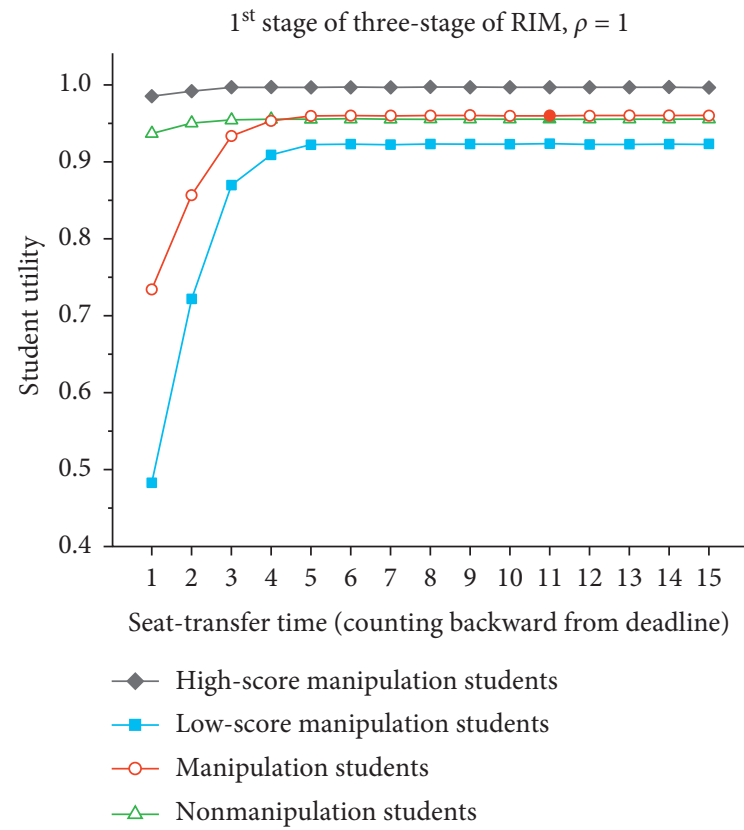

(a)

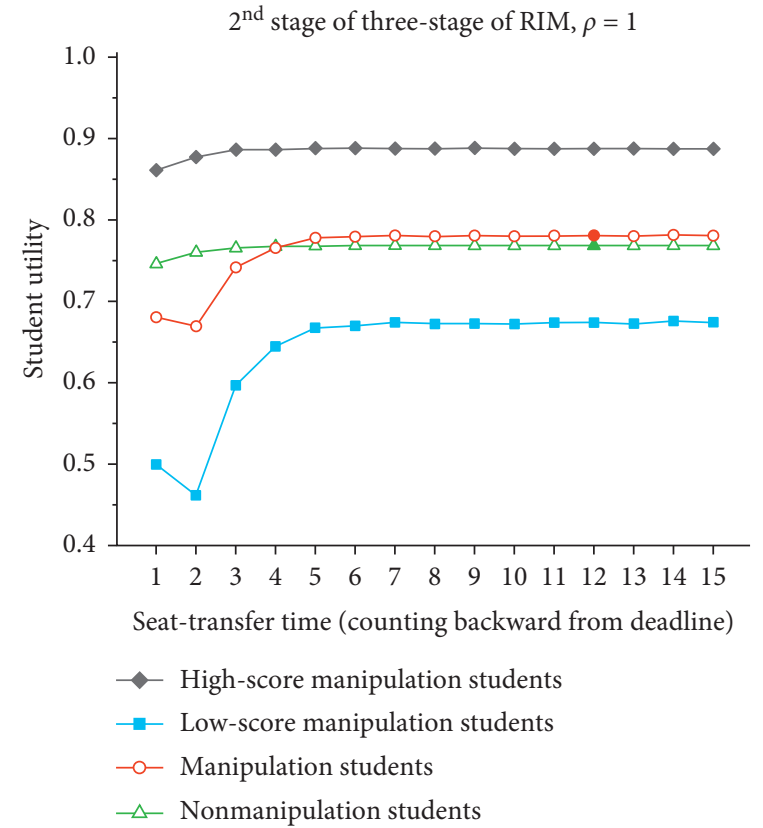

(b)

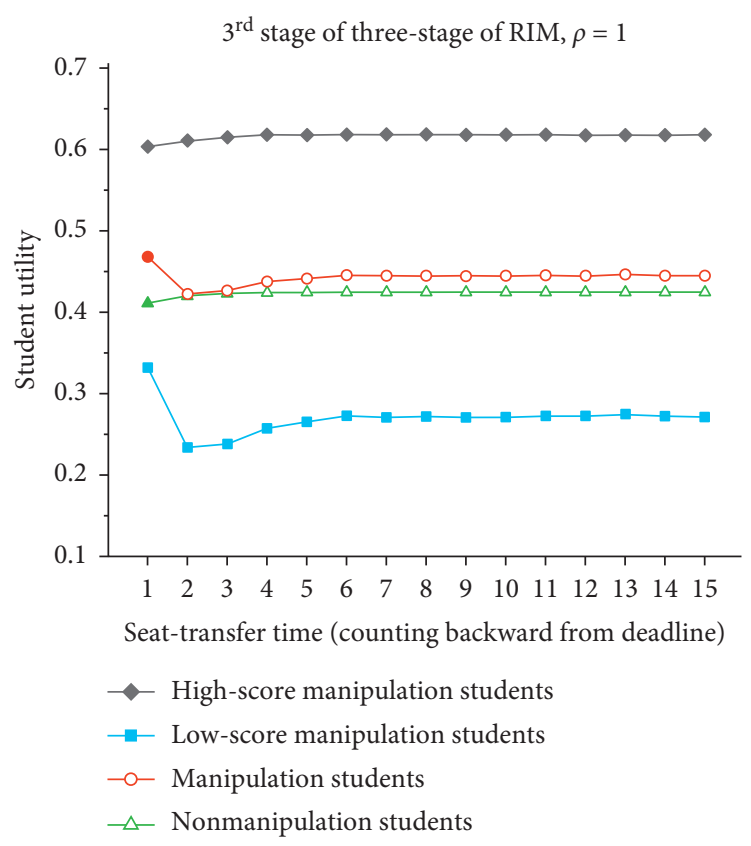

(c)

Figure 6: The utilities of manipulation students and nonmanipulation students when the manipulation students transfer the seat at different stages.

MS-RIM. Take four-stage RIM, for instance, the utility differences are $0.0007,0.0018,0.0154$, and 0.0887 for the 1 st, $2 \mathrm{nd}, 3 \mathrm{rd}$, and 4 th stage. The reasons for this result are twofold. First, when the students conduct coordinating manipulation at any stage, they have the probability that the application for new universities will be unsuccessful due to the network congestion or operation failure. Since the students in the early stages have higher scores, they can usually be admitted by good universities due to their score advantages and then obtain high utilities, that is, the extra utility from successful manipulation over nonmanipulation is roughly the same for all stages, but the utility loss from unsuccessful manipulation in the early stage is larger than that in the late stage, which makes the expected utility gain from manipulation (both successful and unsuccessful) over nonmanipulation is smaller than that in the late stage. Second, the manipulation students in the late stages usually transfer the seat in the rounds closer to the deadline of the submission, that is, the optimal seat-transfer time $\left(t^{*}\right.$ seattransfer) becomes smaller in late stages, as shown in the fourth 
TABLE 1: Simulation results of different versions of RIM.

\begin{tabular}{|c|c|c|c|c|c|c|c|c|c|}
\hline $\begin{array}{l}\text { Version of } \\
\text { RIM }\end{array}$ & $\begin{array}{c}\text { Stage } \\
\text { number }\end{array}$ & $\rho^{*}$ & $\begin{array}{l}t^{*} \text { seat- } \\
\text { transfer }\end{array}$ & $\begin{array}{c}\text { Utility } \\
\text { difference }\end{array}$ & $\begin{array}{c}\text { Manipulation } \\
\text { number }\end{array}$ & $\begin{array}{c}\text { Successful manipulation } \\
\text { number (rate) }\end{array}$ & $\begin{array}{l}\text { Student } \\
\text { welfare }\end{array}$ & $\begin{array}{l}\text { University } \\
\text { welfare }\end{array}$ & $\begin{array}{c}\text { Fairness } \\
\text { index }\end{array}$ \\
\hline $\begin{array}{l}\text { One-stage } \\
\text { RIM }\end{array}$ & Stage 1 & 0.5 & 12 & 0 & 170.1 & $15.94(0.09)$ & 0.7187 & 0.5011 & 0.9458 \\
\hline Two-stage & Stage 1 & 1 & 13 & 0.0031 & 53.22 & $14.83(0.28)$ & 0.7184 & 0.5011 & 0.9282 \\
\hline RIM & Stage 2 & 1 & 11 & 0.0037 & 54.62 & $11.18(0.20)$ & & & \\
\hline \multirow{4}{*}{$\begin{array}{l}\text { Three-stage } \\
\text { RIM }\end{array}$} & Stage 1 & 1 & 11 & 0.0047 & 17.12 & $4.31(0.25)$ & 0.7157 & 0.4988 & 0.793 \\
\hline & Stage 2 & 1 & 12 & 0.0125 & 17.40 & $2.76(0.19)$ & & & \\
\hline & Stage 3 & 1 & 1 & 0.0564 & 17.28 & $9.27(0.54)$ & & & \\
\hline & Stage 1 & 1 & 12 & 0.0007 & 6.48 & $2.61(0.40)$ & 0.7178 & 0.5004 & 0.8743 \\
\hline \multirow{3}{*}{$\begin{array}{l}\text { Four-stage } \\
\text { RIM }\end{array}$} & Stage 2 & 1 & 12 & 0.0018 & 5.77 & $4.46(0.77)$ & & & \\
\hline & Stage 3 & 1 & 10 & 0.0154 & 6.40 & $5.58(0.87)$ & & & \\
\hline & Stage 4 & 1 & 1 & 0.0887 & 6.08 & $3.40(0.56)$ & & & \\
\hline \multirow{5}{*}{$\begin{array}{l}\text { Five-stage } \\
\text { RIM }\end{array}$} & Stage 1 & 1 & 12 & 0.0001 & 1.82 & $1.23(0.68)$ & 0.7196 & 0.5009 & 0.9507 \\
\hline & Stage 2 & 1 & 11 & 0.0083 & 2.10 & $1.99(0.95)$ & & & \\
\hline & Stage 3 & 1 & 6 & 0.0327 & 1.94 & $0.62(0.32)$ & & & \\
\hline & Stage 4 & 1 & 7 & 0.0560 & 2.12 & $1.95(0.92)$ & & & \\
\hline & Stage 5 & 1 & 1 & 0.0904 & 2.18 & $1.59(0.73)$ & & & \\
\hline \multirow{6}{*}{$\begin{array}{l}\text { Six-stage } \\
\text { RIM }\end{array}$} & Stage 1 & 1 & 3 & 0.0017 & 0.52 & $0.47(0.90)$ & 0.7198 & 0.5010 & 0.9841 \\
\hline & Stage 2 & 1 & 4 & 0.0192 & 0.44 & $0.25(0.57)$ & & & \\
\hline & Stage 3 & 1 & 5 & 0.0338 & 0.68 & $0.50(0.74)$ & & & \\
\hline & Stage 4 & 1 & 4 & 0.0344 & 0.58 & $0.44(0.76)$ & & & \\
\hline & Stage 5 & 1 & 1 & 0.0376 & 0.52 & $0.47(0.90)$ & & & \\
\hline & Stage 6 & 1 & 1 & 0.0542 & 0.58 & $0.37(0.64)$ & & & \\
\hline
\end{tabular}

column of Table 1. For example, the manipulation students in the fourth stage of the four-stage RIM transfer their seats in the last round before the deadline, i.e., $t^{*}{ }_{\text {seat-transfer }}=1$. The choice of the optimal transfer time is a trade-off between the probability of operation failure and the probability of being pushed out by other students. If the manipulation students transfer their seat early, the operation failure probability will be smaller because they have more rounds to resubmit their applications, while the probability of being pushed out is larger because more students can notice and apply for the university that the low-score manipulation students applied and push the low-score manipulation students out. The consequence of operation failure is that the students obtain no utility at all, even the normal utility if they did not conduct manipulation. The consequence of being crowded out is that they cannot obtain the extra utility that the manipulation behavior provides. In the late stage, the students have low scores and their utility is usually small if they do not conduct coordinating manipulation; hence, their utility loss when being crowded out is relatively small. Therefore, they will transfer their seats in the round closer to the deadline to decrease the probability of being crowded out to enjoy the extra utility from manipulation. And this result can be summarized as follows.

Result 4. Under a certain version of MS-RIM, the students have a stronger manipulation incentive in late stages than early stages; the manipulation students in late stages transfer their seats at the time closer to the deadline.

In summary, the multistage policy has complex effects on matching results of RIM. The direct effect is that it can reduce the opportunity for the students to coordinate, for example, in the two-stage RIM a student with a score 700 in the first stage cannot hold a seat for the student with a score 550 in the second stage because their deadlines to submit applications are different. This is called the manipulation opportunity reduction effect of the multistage policy. The multistage policy has an indirect effect that more students are willing to conduct coordinating manipulation, and the reason is that the utility from manipulation becomes higher when there are fewer students who can conduct manipulation, which lures more student to adopt the coordinating manipulation, for example, from $\rho^{*}=0.5$, in one-stage RIM to $\rho^{*}=1$ in all other versions of RIM. We call this effect the manipulation incentive amplification effect of the multistage policy. Combining these two effects, there are fewer and fewer students who will conduct coordinating manipulation as the stage numbers increases, as shown in the sixth column of Table 1 and illustrated in Figure 7(a). Furthermore, the multistage policy has another indirect effect, named manipulation success-rate improvement effect. In the one-stage RIM, all manipulation students transfer the seat from the high-score students to low-score students before the deadline and many manipulation students may be pushed out of the admission list of the applied university by other students and fail in the manipulation. However, under RIM with multistage, the number of manipulation students who simultaneously transfer their seats is smaller because of the different deadlines, and there are few switches of the applied university of manipulation students before the deadline, the probability of being pushed out will be smaller, so the success rate of manipulation becomes higher, as shown in the seventh column of Table 1 and illustrated in Figure 7(a). Therefore, the successful manipulation number (the number 


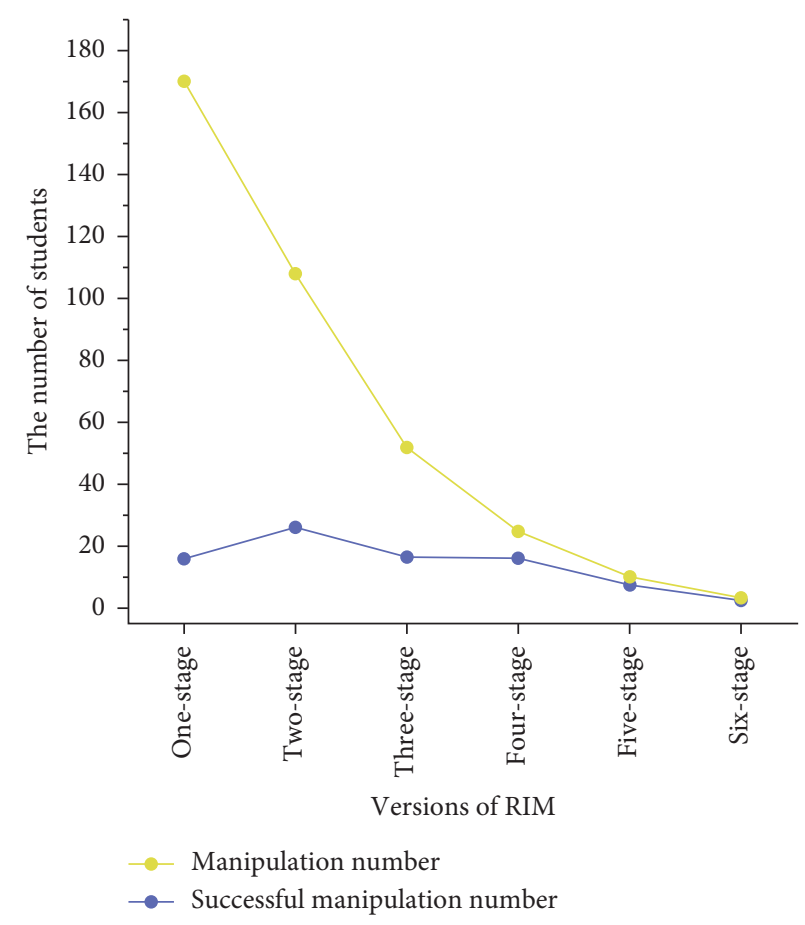

(a)

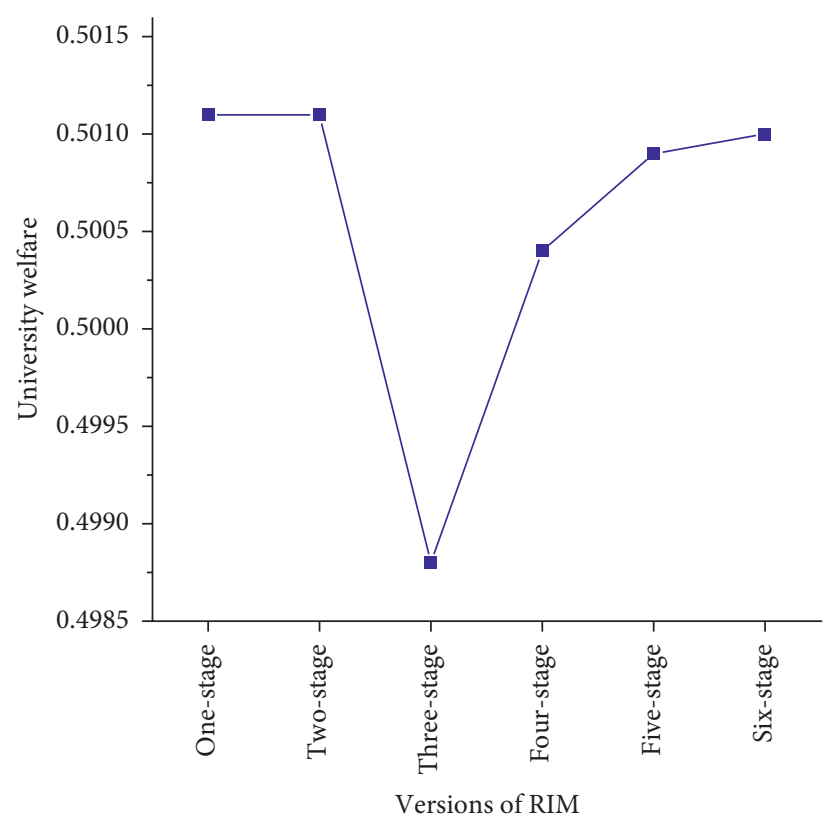

(c)

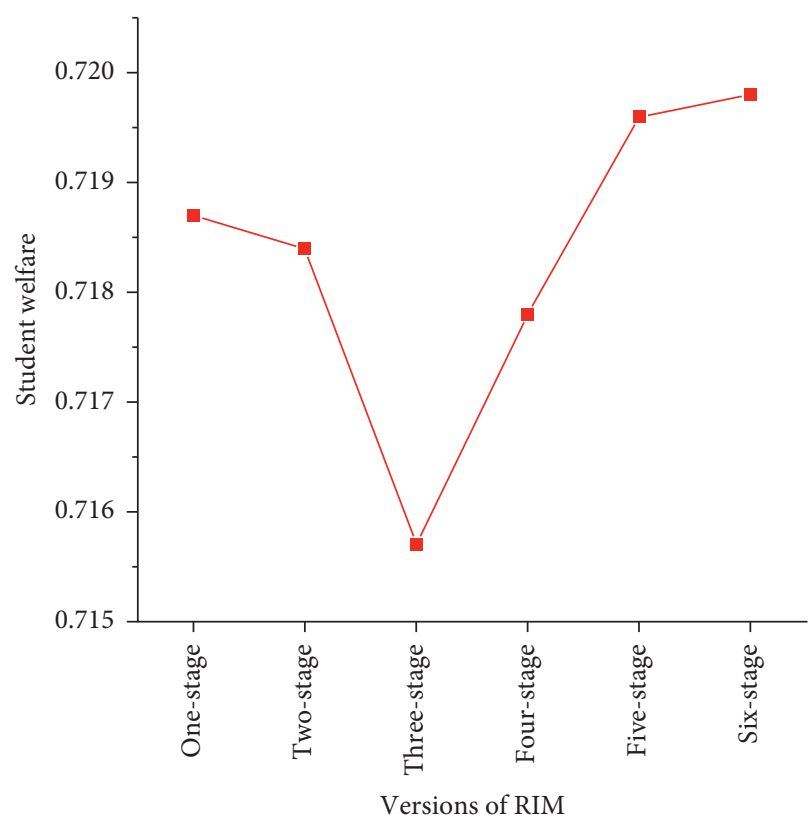

(b)

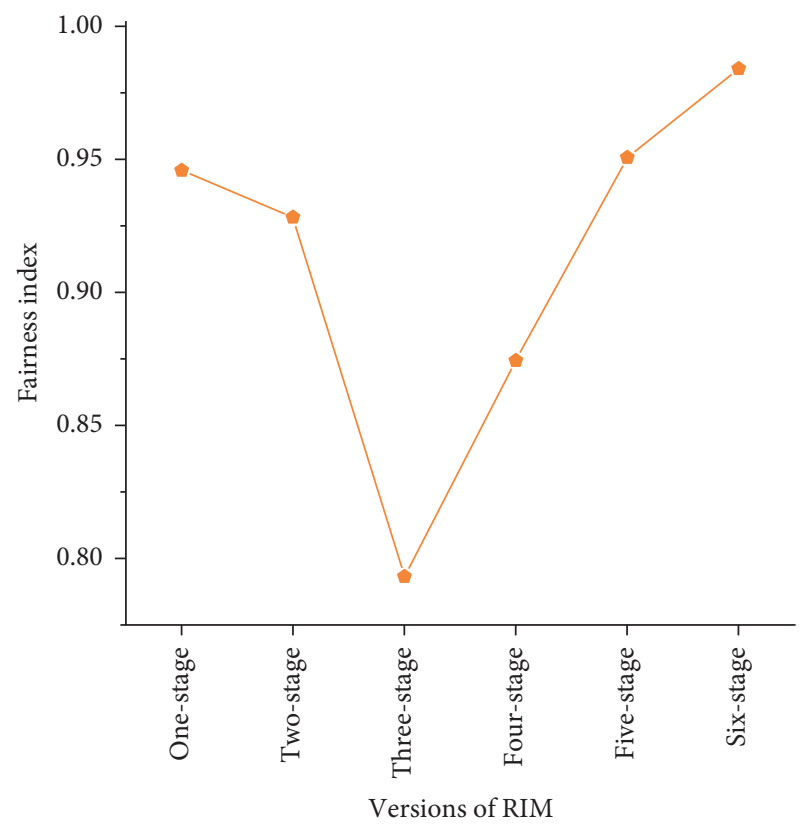

(d)

FiguRE 7: Simulation results under different versions of RIM.

of the students who conduct coordinating manipulation and successfully apply to the intended universities with no operation failure or being pushed out by other students) in two-stage, three-stage, and four-stage RIM are larger than one-stage RIM because the manipulation incentive amplification effect and manipulation success-rate improvement effect dominate the manipulation opportunity reduction effect when the stage number is small; the successful manipulation number drops down in five-stage and six-stage
RIM because the manipulation opportunity reduction effect dominates.

The student welfare (the average utility of all students) decreases slightly under two-stage RIM than one-stage RIM, drops dramatically under three-stage RIM, recovers under four-stage RIM to about the level of one-stage RIM, and keeps increasing under five- and six-stage RIM, as shown in the eighth column of Table 1 and illustrated in Figure 7(b). This pattern is mainly determined by the number of 
successful manipulations under different versions of RIM, and the larger is the successful manipulation number, the smaller is the student welfare. The sudden decrease under three-stage RIM is also on account of the optimal seattransfer time in the third stage is the last round before the deadline, many students (both manipulation and nonmanipulation students) are pushed out of admission lists and are not matched to any university and obtain zero utility.

The university welfare changes in the similar pattern of the student welfare as the state number of MS-RIM varies, as shown in the ninth column of Table 1 and illustrated in Figure $7(\mathrm{c})$. Because all universities have the identical preference to students according to their scores, the average university utility does not change no matter how the students and universities are matched as long as the quotas of all universities are fulfilled, and hence the university welfare is the same for one-stage and two-stage RIM because manipulation students transfer their seats very early and almost no student is pushed out at the last minute before the deadline under these two mechanisms. Under the threestage RIM, however, all manipulation students in the third stage transfer their seats in the last round before the deadline, and many students are pushed out of admission lists of their applied universities and have no chance to resubmit their applications; hence, some universities cannot fulfill their enrolment quota and then obtain smaller utilities. The university welfare reduction under the four-, five-, and six-stage RIM is also due to the unfulfilled quota of universities, but the welfare reduction becomes smaller as the stage number increases thanks to the decline of the successful manipulation number.

The fairness index changes in a similar pattern, as shown in the ninth column of Table 1 and illustrated in Figure 7(d). More students feel unfair under the two-, three-, and fourstage RIM than under one-stage RIM, and fewer students are feeling unfair under five- and six-stage RIM. It can be expected that if there are enough many stages in an MS-RIM, the maximal score difference of the students in one stage will be smaller than the critical value for coordinating manipulation (score_diff) and there will be no coordinating manipulation anymore, and the social welfare and fairness reaches the maximum (equal to the value of the state with no manipulation $(\rho=0)$ in Figure 3$)$. The results of the manipulation number, social welfare, and fairness are summarized as follows.

Result 5. Under MS-RIM with a small stage number, more students successfully conduct coordinating manipulation than one-stage RIM, and the social welfare and fairness are diminished; under MS-RIM with a large stage number, there are fewer successful manipulation students than one-stage RIM, and the social welfare and fairness are improved.

\section{Conclusions}

Matching plays an important role in several processes in our social system, such as dating, marriage, hiring, recommending, and more [26-30]. Here, we investigate another instance of matching: the college application and admission. The development of information and communication technology makes it possible for the college admission process to be automated online. The innovation of the matching algorithms in cyberspace can dramatically improve the efficiency of the corresponding process in physical space.

This paper studies the manipulation behaviors in the real-time interactive mechanism (RIM) recently adopted in Inner Mongolia of China and several other places globally. This mechanism has many advantages, such as transparency and real-time feedback. However, it is vulnerable to manipulation by the coordination of students. A high-score student can perform a last-minute switch on the university applied, opening a slot for a student with a much lower score. And this erodes the social welfare and fairness of the admission system.

Using the approach of agent-based modeling, we study the manipulation behavior in the real-time interactive mechanism. It is found that the benefit from manipulation is positive when there are few manipulation students but negative when manipulation occurs too frequently. Hence, a stable state where manipulation and nonmanipulation students have equal utility characterizes the outcome of the students' strategic choices. Simulations show that a large portion of students will choose to perform manipulation at the steady state and social welfare and fairness are damaged.

To cope with this issue, we investigate the multistage RIM (MS-RIM), where students with different ranges of scores are given different deadlines for application modification. By intensive simulations, we identify three effects of the multistage policy. It can reduce the chance of students to coordinate due to the different deadlines to submit applications. Nevertheless, it amplifies the students' incentive to conduct coordinating manipulation due to the scarcity of manipulation opportunity and consequent higher reward. Moreover, it helps to avoid the congestion that all manipulation students transfer their seats almost simultaneously and increases the success rate of the manipulation.

Due to the combined effects of multistage policy, more students conduct coordinating manipulation successfully under MS-RIM with a small number of stages, and the social welfare and fairness are diminished; while under MS-RIM with a larger number of stages, the successful manipulation number decreases and the social welfare and fairness are improved. However, a large number of stages imply that the mechanism needs to be executed for a very long time that may bring inconvenience. As an implication, some other policies are needed to really combat the coordinating manipulation in the real-time interactive mechanism, such as limiting the times that a student can resubmit his application unless he is pushed out of the admission list of the applied university.

\section{Data Availability}

The data used to support the findings of the study are available from the corresponding author upon request. 


\section{Conflicts of Interest}

The authors declare that there are no conflicts of interest regarding the publication of this paper.

\section{Acknowledgments}

This work was supported partly by the National Natural Science Foundation of China (71201129 and 71561007), Fundamental Research Funds for the Central Universities (XDJK2016B008), Joint Fund Project in Science and Technology Department of Guizhou Province [Qian Ke He LH Zi [2017] 7001], and major projects of Natural Science Innovation Corps in Education Department of Guizhou Province [Qian Jiao He KY Zi [2017] 051].

\section{References}

[1] D. Gale and L. S. Shapley, "College admissions and the stability of marriage," The American Mathematical Monthly, vol. 69, no. 1, pp. 9-15, 1962.

[2] A. Abdulkadiroglu and T. Sonmez, "School choice: a mechanism design approach," American Economic Review, vol. 93, no. 3, pp. 729-747, 2003.

[3] Y. Chen and O. Kesten, "Chinese college admissions and school choice reforms: a theoretical analysis," Journal of Political Economy, vol. 125, no. 1, pp. 99-139, 2017.

[4] M. Zhu, "College admissions in China: a mechanism design perspective," China Economic Review, vol. 30, pp. 618-631, 2014.

[5] F. Li, "Economic thinking of the college admissions in China: comparison of the immediate acceptance mechanism, parallel mechanism and real-time dynamic mechanism," Educational Research at Tsinghua University, no. 5, pp. 48-55, 2011.

[6] P. A. Pathak, "The mechanism design approach to student assignment," Annual Review of Economics, vol. 3, no. 1, pp. 513-536, 2011.

[7] H. F. Nie, "A game-theoretical analysis of China's college admission mechanism," China Economic Quarterly, vol. 6, no. 3, pp. 899-916, 2007, in Chinese.

[8] A. Abdulkadiroglu, P. A. Pathak, A. E. Roth, and T. Sonmez, "The Boston public school match," American Economic Review, vol. 95, no. 2, pp. 368-371, 2005.

[9] H. Ergin and T. Sonmez, "Games of school choice under the Boston mechanism," Journal of Public Economics, vol. 90, no. 1-2, pp. 215-237, 2006.

[10] P. A. Pathak and T. Sonmez, "School admissions reform in chicago and england: comparing mechanisms by their vulnerability to manipulation," American Economic Review, vol. 103, no. 1, pp. 80-106, 2011.

[11] P. A. Pathak and T. Sönmez, "Leveling the playing field: sincere and sophisticated players in the Boston mechanism," American Economic Review, vol. 98, no. 4, pp. 1636-1652, 2008.

[12] Y. Chen and O. Kesten, "Chinese college admissions and school choice reforms: an experimental study," Games and Economic Behavior, vol. 115, pp. 83-100, 2019.

[13] G. Y. Shi, Y. X. Kong, B. L. Chen, G. H. Yuan, and R. J. Wu, "Instability in stable marriage problem: matching unequally numbered men and women," Complexity, vol. 2018, Article ID 7409397, 2018.
[14] Y. X. Kong, G. H. Yuan, L. Zhou, R. J. Wu, and G. Y. Shi, "Competition may increase social utility in bipartite matching problem," Complexity, vol. 2018, Article ID 4092056, 2018.

[15] G. Haeringer and F. Klijn, "Constrained school choice," Journal of Economic Theory, vol. 144, no. 5, pp. 1921-1947, 2009.

[16] R. M. Antonio, "Implementation of stable solutions in a restricted matching market," Review of Economic Design, vol. 3, no. 2, pp. 137-147, 1998.

[17] C. Calsamiglia, G. Haeringer, and F. Klijn, "Constrained school choice: an experimental study," American Economic Review, vol. 100, no. 4, pp. 1860-1874, 2010.

[18] I. Bo and R. Hakimov, The Iterative Deferred Acceptance Mechanism, SSRN, Rochester, NY, USA, 2018.

[19] I. Bo and R. Hakimov, "Iterative versus standard deferred acceptance: experimental evidence," The Economic Journal, vol. 130 , no. 626 , pp. 356-392, 2020.

[20] F. Klijn, J. Pais, and M. Vorsatz, "Static versus dynamic deferred acceptance in school choice: theory and experiment," Games and Economic Behavior, vol. 113, pp. 147-163, 2019.

[21] Inner Mongolia Admissions and Exam Infor-Net. Notice on the 2020 Inner Mongolia Gaokao College Application Submission. https://www.nm.zsks.cn/ptgxzs/ggl/202007/ t20200720_40198.html.

[22] J. M. Epstein, "Agent-based computational models and generative social science," Complexity, vol. 4, no. 5, pp. 41-60, 1999.

[23] S. H. Chen, Agent-Based Computational Economics: How the Idea Originated and where It is Going, Routledge, New York, NY, USA, 2017.

[24] S.-H. Chen, C. H. Wang, and W. Chen, "Matching impacts of school admission mechanisms: an agent-based approach," Eastern Economic Journal, vol. 43, no. 2, pp. 217-241, 2017.

[25] C. H. Wang, B.-T. Chie, and S.-H. Chen, "Transitional student admission mechanism from tracking to mixing: an agentbased policy analysis," Evolutionary and Institutional Economics Review, vol. 14, no. 1, pp. 253-293, 2017.

[26] E. E. Bruch and M. E. J. Newman, "Aspirational pursuit of mates in online dating markets," Science Advances, vol. 4, no. 8, 2018

[27] Y. Xie, S. Cheng, and X. Zhou, "Assortative mating without assortative preference," Proceedings of the National Academy of Sciences, vol. 112, no. 19, pp. 5974-5978, 2015.

[28] T. Jia, R. F. Spivey, B. Szymanski, and G. Korniss, "An analysis of the matching hypothesis in networks," PLoS One, vol. 10, no. 6,2015

[29] P. Haller and D. F. Heuermann, "Job search and hiring in local labor markets: spillovers in regional matching functions," Regional Science and Urban Economics, vol. 60, pp. 125-138, 2016.

[30] L. Lü, C. H. Yeung, Y.-C. Zhang, Z.-K. Zhang, and T. Zhou, "Recommender systems," Physics Reports, vol. 519, no. 1, pp. 1-49, 2012. 